\title{
Plasmonic Metamaterials and Nanocomposites with the Narrow Transparency Window Effect in Broad Extinction Spectra
}

\author{
Hui Zhang, ${ }^{\dagger}$ Hilmi Volkan Demir, ${ }^{\ddagger} \S$ and Alexander O. Govorov* ${ }^{\dagger}$ \\ ${ }^{\dagger}$ Department of Physics and Astronomy, Ohio University, Athens, Ohio 45701, United States \\ ${ }^{\ddagger}$ Department of Electrical and Electronics Engineering, Department of Physics, UNAM - Institute of Materials Science and \\ Nanotechnology, Bilkent University, Ankara 06800, Turkey \\ ${ }^{\S}$ School of Electrical and Electronic Engineering, School of Physical and Mathematical Sciences, Nanyang Technological University, \\ Nanyang Avenue, Singapore 639798, Singapore
}

\section{Supporting Information}

\begin{abstract}
We propose and describe plasmonic nanomaterials with unique optical properties. These nanostructured materials strongly attenuate light across a broad wavelength interval ranged from $400 \mathrm{~nm}$ to $5 \mu \mathrm{m}$ but exhibit a narrow transparency window centered at a given wavelength. The main elements used in our systems are nanorods and nanocrosses of variable sizes. The nanomaterial can be designed as a solution, nanocomposite film or metastructure. The principle of the formation of the transparency window in the broad extinction spectrum is based on the narrow lines of longitudinal plasmons of single nanorods and nanorod complexes. To realize the spectrum with a transmission window, we design a nanocomposite material as a mixture of nanorods of different sizes. Simultaneously, we exclude nanorods of certain lengths from the nanorod ensemble. The width of the plasmonic transparency window is determined by the intrinsic and radiative broadenings of the nanocrystal plasmons. Nanocrystals can be randomly dispersed in a solution or arranged in metastructures. We show that interactions between nanocrystals in a dense ensemble can destroy the window effect and, simultaneously, we design the metastructure geometries with weak destructive interactions. We also describe the effect of narrowing of the transparency window with increasing the concentration of nanocrystals. Two well-established technologies can be used to fabricate such nanoand metamaterials, the colloidal synthesis, and lithography. Nanocomposites proposed here can be used as optical materials and smart coatings for shielding of electromagnetic radiation in a wide spectral interval with a simultaneous possibility of communication using a narrow transparency window.
\end{abstract}

KEYWORDS: metamaterial, optical properties, transparency, plasmon, nanocomposite, nanorod, nanowire

$\mathrm{M}$ etal nanocrystals (NCs) and metamaterials with strong plasmonic resonances in the visible and infrared spectral intervals often exhibit very unique optical properties. ${ }^{1,2}$ In single nanocrystals with specially designed shapes, plasmonic resonances can be efficiently tuned with the geometry and can be made very strong and narrow. ${ }^{3-5}$ Especially these properties are characteristic for the plasmonic nanorods (NRs) that exhibit strong and narrow longitudinal resonances. ${ }^{4}$ Whereas nanospheres, nanorods, and nanocubes with small sizes can be grown using a colloidal synthesis in solution, ${ }^{3}$ larger-size nanostructures are conveniently fabricated by lithographic methods. ${ }^{6}$ Lithographically made $2 \mathrm{D}$ and $3 \mathrm{D}$ metamaterials employ electromagnetic interactions between building blocks to create interesting optical responses. One of the prominent effects coming from the interactions between single nanocrystals is the Fano effect. This effect can occur in a purely plasmonic system ${ }^{7-10}$ or in hybrid exciton-plasmon nanostructures. ${ }^{11-14}$ The Fano effect typically originates from an interaction between broad and narrow resonances in a system composed of two or several elements. Another phenomenon, which is relevant to the plasmonic Fano effect, is the plasmoninduced transparency in planar metamaterials composed of a few interacting nanocrystals. ${ }^{15-17}$ One more mechanism for a formation of spectral windows is an extraordinary optical transmission in plasmonic nanohole arrays where transmission windows are attributed to the presence of constructive interference of surface plasmonic waves. ${ }^{18}$ The extraordinary optical transmission is an angle-dependent effect. Here we propose another approach to the effect of transparency window in nanostructured systems. Our approach is to construct a nanomaterial as a composition of single nanocrystals and nanocrystal complexes with narrow and tunable absorption lines.

Received: April 2, 2014

Published: August 1, 2014 
a)

3D media: Solution or film

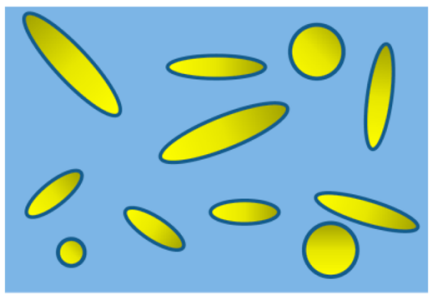

b)

Layer-by-layer deposition film c)

Planar metastructure

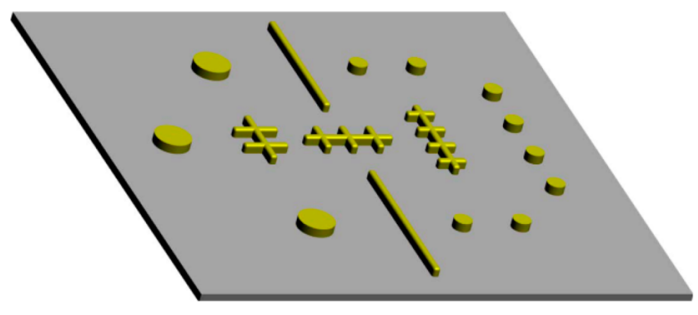

Figure 1. Models of the nanostructured systems discussed in the paper. (a, b) Composite media incorporating plasmonic nanorods and nanoparticles built into polymer films and solutions. (c) Planar metastructure composed of nanorods, nanocrosses, and nanodisks. This metastructure is designed to exhibit the transparency window effect.

Here we describe plasmonic media with very unusual optical properties. The idea is to design a medium or metamaterial that should attenuate light in a very broad spectral interval but exhibit a narrow transparency window at a given wavelength. Such optical materials can be used for electromagnetic shielding that simultaneously permits communications via the transparency window. Looking at the available materials and media, we can see that the optical materials found around us may exhibit narrow absorption lines or absorption bands. For example, dye molecules typically demonstrate narrow absorption lines in the visible, or water molecules exhibit a set of narrow absorption lines in the infrared. Or, another material system widely available is amorphous carbon and carbon particles that strongly absorb and scatter light in a wide spectral interval without any transparency intervals. A medium with the needed properties, that is, with a broad extinction spectrum and a narrow transmission window, seems to be challenging to realize. Nevertheless, we identify theoretically the materials and designs that may lead to a realization of such media. Importantly, the media proposed in this paper are based on the real materials and realistic technologies. A narrow transparency window in a very broad extinction spectrum can exist in nanocomposites and metamaterials incorporating nanorods, nanoparticles (NPs) and nanocrosses (Figure 1). The components and composition of these optical media should be carefully designed. The elements of the nanocomposite can be as simple as single nanorods and nanoparticles (Figure 1a,b) or more complex, such as nanocrosses and multibar nanocrosses (Figure 1c). The main building block of the proposed systems is a nanorod with strong, narrow and tunable extinction lines of longitudinal plasmons. The media with these unique optical spectra can be fabricated as dispersions, films, coatings, planar metamaterials, and so on. Nanocrystals in such media can be either randomly oriented (Figure 1a) or arranged in the parallel layers (Figure 1b,c). In the cases of solution or polymer film, nanocrystals are randomly oriented, and correspondingly, optical properties of single nanocrystals become averaged over all orientations. In the polymer systems, nanorods can be arranged in layers by the layer-by-layer deposition method, which will make the system optically anisotropic (Figure 1b). Lithography is a very powerful method that allows us to fabricate custom-designed nanocrystals and metastructures. In our calculations, we observed that interactions between nanocrystals in densely packed compositions tend to destroy the window effect. Therefore, we computationally designed nanocrosses and metastructures in which destructive interactions are weak and the spectral transparency widow is well-defined (Figure 1c). Such nanocrosses and metastructures can be fabricated by lithography. As an example, Figure 1c shows a metastructure featuring the transparency window effect in the IR region. Below we will describe the principles to design such optical materials.

To the best of our knowledge, isotropic optical materials with a very broad extinction spectrum featuring a narrow window do not exist yet, but can be fabricated in the ways described here. Such nanomaterials can find applications such as smart coatings and screens for shielding of electromagnetic radiation. The principle differences between our structures and the exciting planar nanostructures, which can also be used for the window effect, are in the anisotropy and the interference effects. Planar Bragg reflectors and metamaterials exhibit strongly anisotropic transmission spectra, whereas our nanocomposites are isotropic. Bragg-reflector filters and planar metamaterials featuring transmission bands are based on an electromagnetic interference and interactions between their elements, whereas our structures are composed of weakly interacting elements since, as we have shown, strong interactions can destroy the window effect. Moreover, the designed metrastructure complexes may have simultaneously densely packed elements and weak interactions between these elements. The width of the narrow feature in the spectra is determined by the intrinsic and radiative broadenings of the plasmon peaks and by the composition of a metastructure or a metasolution. Since our structures exhibit narrow spectral features in very broad extinction spectra, such nanomaterials should be designed carefully and, therefore, the role of modeling is crucial.

The paper is organized in the following way. The discussion develops from the simple to the complex. The first sections concern composite media made from single plasmonic nanorods of small sizes, whereas the following sections describe 


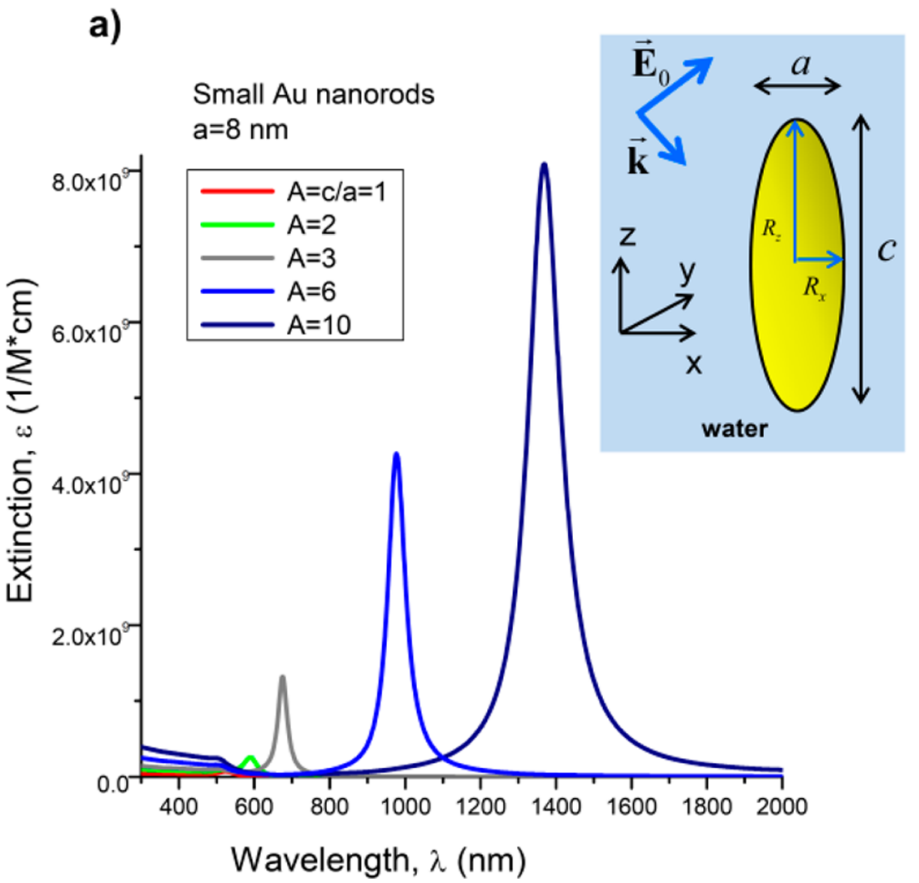

b)
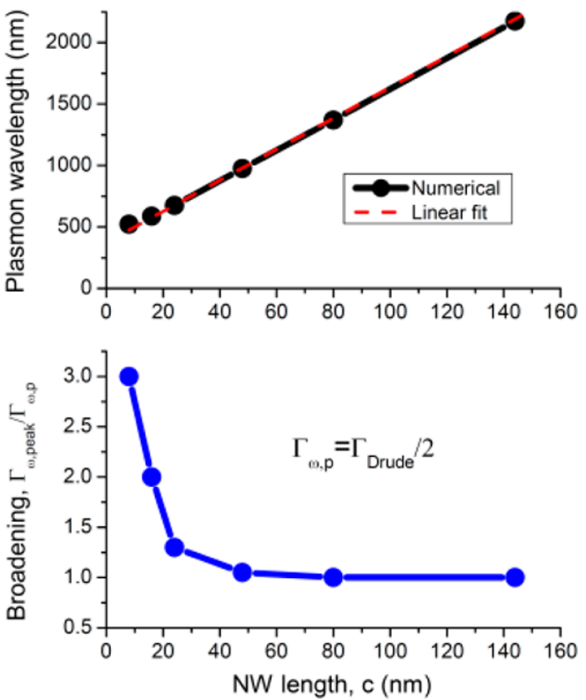

Figure 2. (a) Extinction spectra of small Au nanorods for various aspect ratios. The width of NRs is kept constant, $a=2 \times R_{x}=8 \mathrm{~nm}$. The extinctions were averaged over the orientation of incident electric field. (b) Wavelength and broadening of the L-plasmon peak as a function of the NR length.

media incorporating large nanorods, nanocrosses, and metastructures.

\section{SMALL NONINTERACTING PLASMONIC NANORODS}

Optical Properties of Small NRs. We start with the small plasmonic Au nanorods exhibiting narrow plasmon resonances. Optical properties of these nanocrystals are described by the analytical theory. ${ }^{19,20}$ The extinction cross-section of a small anisotropic NC is given by its absorption rate:

$$
\sigma_{\alpha}=\frac{Q_{\alpha}}{I_{0}}=\frac{2 \pi}{c_{0} \sqrt{\varepsilon_{0}} \cdot E_{0}^{2}} Q_{\alpha}
$$

where $Q_{\alpha}$ and $I_{0}$ are the absorption rate and the incident photon flux, respectively; $E_{0}$ is the amplitude of incident electromagnetic field and $\varepsilon_{0}$ is the dielectric constant of matrix; the index $\alpha$ describes the direction of the electric field in the incident wave and $\alpha=x, y, z$ (Figure 2). For the model of plasmonic nanorod, we choose an elongated ellipsoid with $R_{x}=$ $R_{y}<R_{z}$, where $R_{\alpha}$ is the NR radius in the $\alpha$-direction and again $\alpha=x, y, z$. Simultaneously we adopt the notations for the length and width of NR, $a=2 R_{x}=2 R_{y}$ and $c=2 R_{z}$ (Figure 2). Equations to compute the absorption cross-section of small ellipsoids are well-known ${ }^{19,20}$ and can be found in Supporting Information. In a 3D medium incorporating randomly oriented NRs, the cross-section should be averaged over the orientations,

$$
\sigma=\frac{\sigma_{x}+\sigma_{y}+\sigma_{z}}{3}
$$

Sometimes it is more convenient to use, instead of the extinction cross section, a molar extinction coefficient defined as

$$
\varepsilon_{\left[(\mathrm{M} \cdot \mathrm{cm})^{-1}\right]}=N_{\mathrm{A}} \frac{10^{-4}}{0.23} \cdot \sigma_{\left[\mathrm{cm}^{2}\right]} \cdot
$$

The extinction spectrum of a single NR exhibits a strong resonance related to the longitudinal plasmon (L-plasmon) at a longer wavelength and a relatively weak peak for the transverse plasmon at a shorter wavelength (Figure 2a). To realize a material with a narrow transparency window, we will utilize the strong L-plasmon resonance. We therefore look now at the function $\sigma_{z}(\omega)$ and investigate the position and broadening of L-plasmon as a function of the NR aspect ratio

$$
A=\frac{R_{z}}{R_{x}}=\frac{c}{a}
$$

This can be done numerically using the empirical dielectric function. ${ }^{21}$ Or, we can use the Drude and Drude-Lorentz models, ${ }^{22}$ which can be convenient to understand the nature of plasmon-peak broadening (see Supporting Information).

The properties of the L-plasmon peak are summarized in Figure $2 \mathrm{~b}$. To better understand these properties, one can perform analytical calculations starting from the absorption rate $Q_{z}(\omega)$ (see Supporting Information). First, the L-plasmon wavelength is approximately a linear function of the NR ratio and the intensity of the L-plasmon peak rapidly increases with increasing the NR length (Figure 2b). The broadening of the Lplasmon in the spectrum $\sigma_{z}(\omega)$ stays the same for large NR ratios and becomes strongly increased for nearly spherical nanocrystals due to the interband processes. For long NRs, the plasmon-peak width is given by the Drude broadening parameter $\Gamma_{\text {Drude }}$ (Supporting Information). Regarding the absorption cross-section of NRs as a function of the wavelength, $\sigma_{z}(\lambda)$, the broadening of the L-plasmon peak in these spectra increases with increasing the NR aspect ratio as $\Gamma_{p, \lambda}=\left(\Gamma_{\text {Drude }} / 2\right)\left(\lambda_{\mathrm{p}, \mathrm{L}}^{2} / 2 \pi c_{0}\right)$, where $\lambda_{p, L}$ is the L-plasmon wavelength, which is an increasing function of the aspect ratio. 


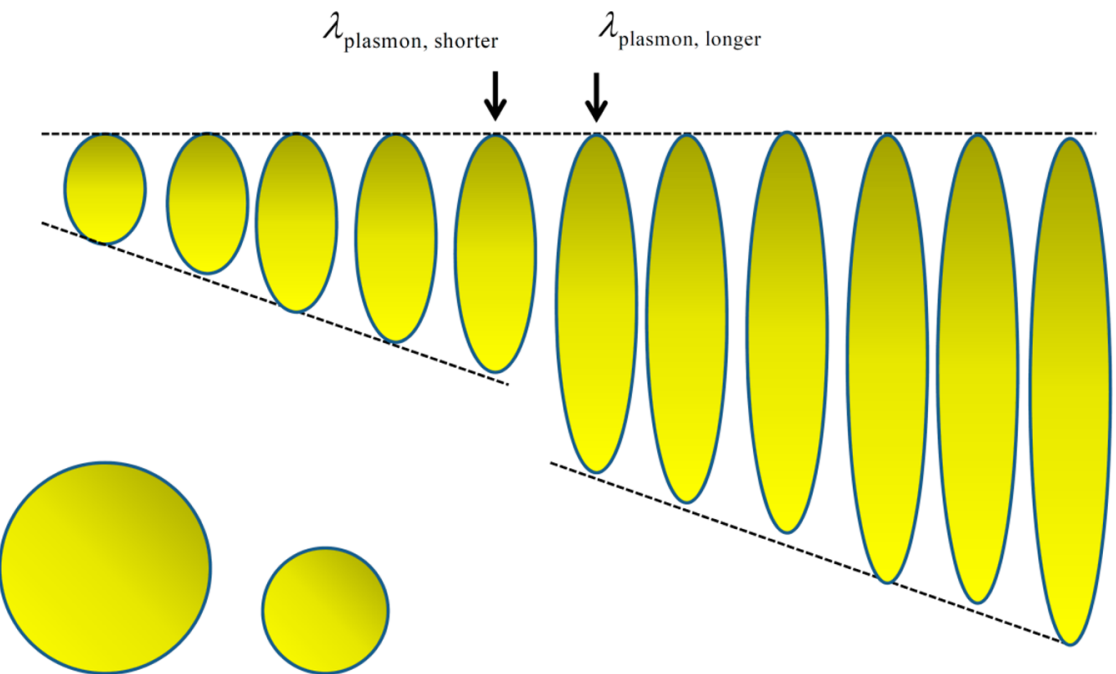

For the $300-700 \mathrm{~nm}$ region

Figure 3. Model of collection of NRs exhibiting a transparency window effect in the transmission spectra. The NR collection does not include NRs with lengths in the interval $50 \mathrm{~nm}<c<70 \mathrm{~nm}$. Correspondingly, the NRs with the gap sizes $(c=50 \mathrm{~nm}$ and $c=70 \mathrm{~nm})$ determine the positions of the edges of the transparency window $\lambda_{\text {plasmon,shorter }}$ and $\lambda_{\text {plasmon,longer }}$. The NR collection can be supplemented with spherical nanoparticles that create strong absorption in the interval $300-700 \mathrm{~nm}$.

The above short discussion on the position and broadening of the L-plasmon in single NRs will be used in the following sections to understand the limits for the spectral width of the transparency window in plasmonic nanomaterials.

\section{COLLECTIONS OF SMALL NRS}

Our first model is a composite medium that incorporates small plasmonic NRs with random positions and orientations. We assume that the ensemble of NRs is diluted and Coulomb and electromagnetic interactions between the NRs are weak and can be neglected. We now construct a medium from a mixture of NRs of various sizes according to simple rules outlined below.

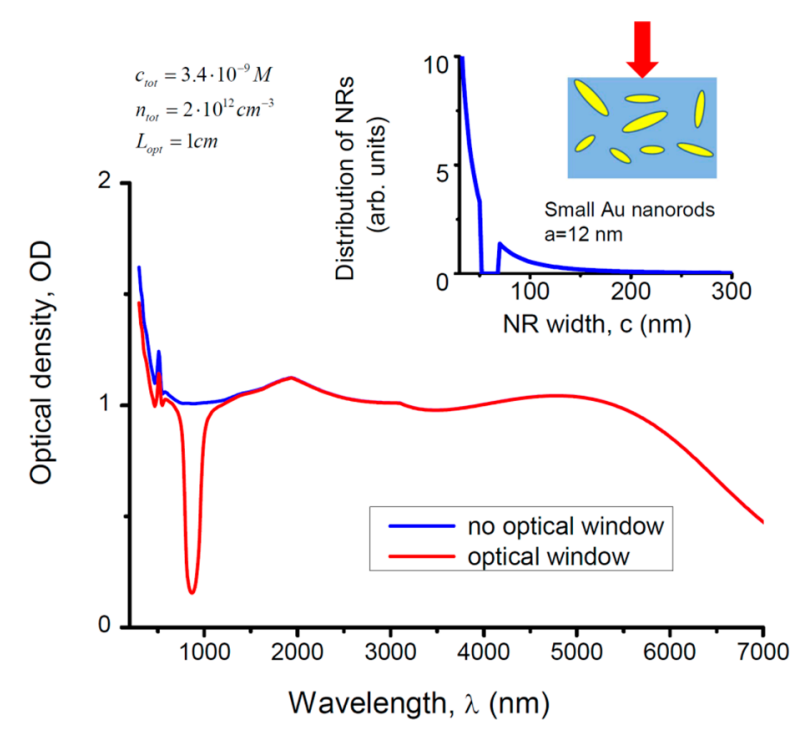

Figure 4. Optical density for the model collections of NRs. Blue and red curves show the absorptions for the NR collections without and with the window effect, respectively. These curves were calculated from the NR distributions given by eqs 4 and 5 . Inset: The NR-size distribution with a gap from 50 to $70 \mathrm{~nm}$. The inset also illustrates a composite medium with randomly orientated NRs.

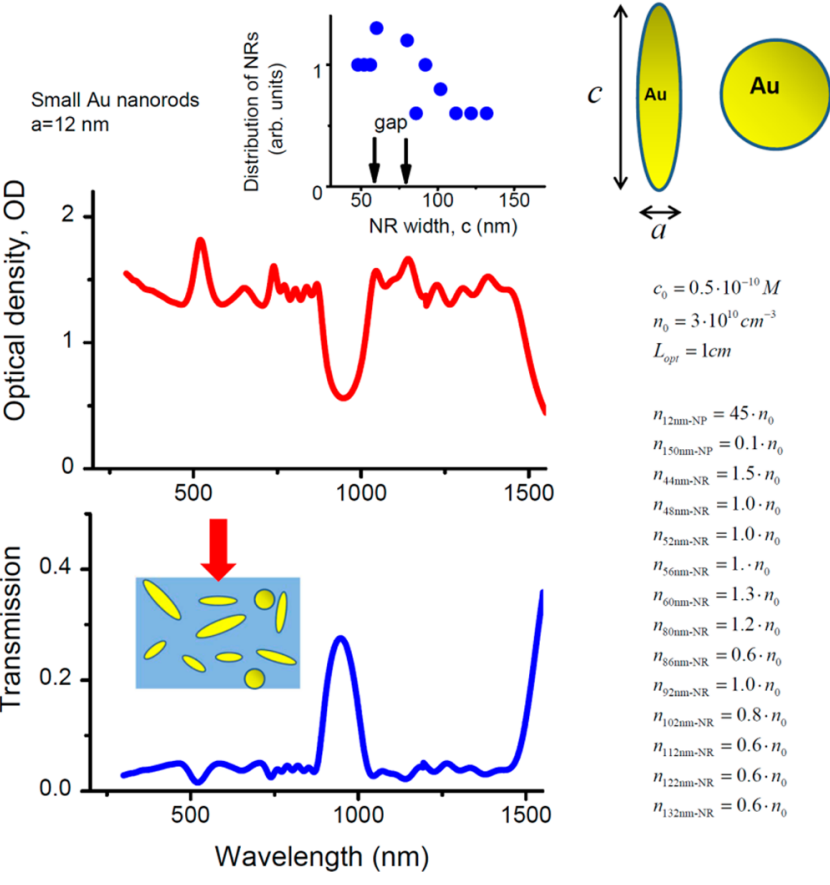

Figure 5. Optical density and transmission of the medium incorporating a discrete size-distribution of NRs. On the right-hand side, we list the NRs and their concentrations. This composite contains also spherical Au NPs of two sizes. Insets show the distribution of NR sizes and the model of plasmonic composite medium. A matrix for this media can be liquid or polymer.

Designing collection of NRs, we do not include nanocrystals with a certain length and, therefore, the transmission spectrum of such composite materials acquires a window centered at a certain wavelength (Figure 3 ). The best transmission windows can be made from an infinite collection of small NRs of various sizes and this collection of NRs should be represented by a continuous size-distribution function. However, practical 

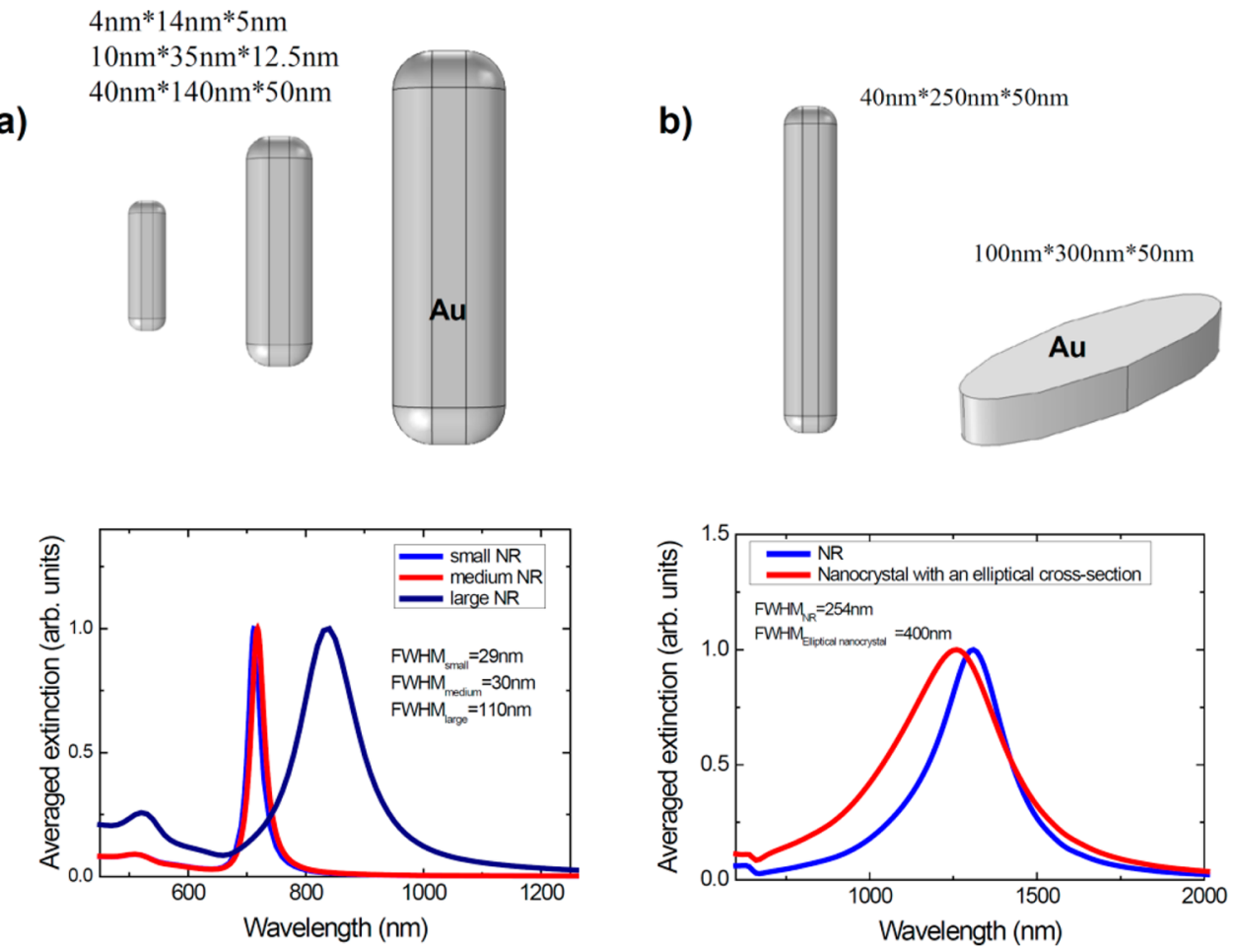

Figure 6. Electrodynamic properties of nanorods and nanodisks. (a) Broadening of the L-plasmon peak strongly increases with a nanocrystal size due to the radiative decay. (b) NRs typically exhibit narrower L-plasmon lines compared to the case of nanodisks with a similar plasmonic wavelength.

implementations of this simple idea will require a discrete sequence of NRs and we will give below related examples.

Continuous Collection of NRs. We now consider a collection of NRs with a constant width $R_{x}=R_{y}$ and a variable length $R_{z}$. A size distribution of NRs is defined by the distribution function $\delta n_{\mathrm{NR}}\left(R_{z}\right)$ that can be normalized to the total density of NRs

$$
n_{\text {tot }}=\int_{R_{z, \min }}^{R_{z, \max }} \mathrm{d} r_{z} \cdot \delta n_{\mathrm{NR}}\left(r_{z}\right)
$$

where $r_{z}$ is the NR $z$-radius, which is a variable in this case. The resulting averaged cross-section of a composite is given by the integral

$$
\bar{\sigma}=\frac{1}{n_{\text {tot }}} \int_{R_{z, \min }}^{R_{z, \max }} \mathrm{d} r_{z} \cdot \delta n_{\mathrm{NR}}\left(r_{z}\right) \cdot \sigma\left(r_{z}\right)
$$

Then the transmitted intensity and the transmission coefficient of a mixture are given by

$$
I_{\mathrm{t}}=I_{0} e^{-\alpha}, \quad T=\frac{I_{\mathrm{t}}}{I_{0}}=e^{-\alpha}=10^{-\mathrm{OD}}
$$

where the exponents, which are the optical densities, should be calculated as

$$
\begin{aligned}
& \alpha=L_{\text {path }} n_{\text {tot }} \bar{\sigma} \equiv L_{\text {path }} \int_{R_{z, \min [\mathrm{nm}]}}^{R_{z, \max [\mathrm{nm}]}} \cdot \delta n_{\mathrm{NR}}\left(r_{z}\right) \cdot \sigma\left(r_{z}\right) \\
& \mathrm{OD}=\frac{\alpha}{\ln [10]}
\end{aligned}
$$

where $L_{\text {path }}$ is the optical path and $\sigma\left(r_{z}\right)$ is the NR absorption cross-section averaged over the orientations (see eq 2 ). In the IR spectral region, the $z$-contribution to the cross-section, $\sigma_{z}\left(r_{z}\right)$, dominates the spectrum due to the very strong L- plasmon response. Now we will try this approach with the following model density distribution

$$
\delta n_{\mathrm{NR}, 0}\left(r_{z}\right)=n_{0} \frac{1}{r_{z[\mathrm{~nm}]}^{2 \cdot 6}}
$$

where $r_{z[\mathrm{~nm}]}=c / 2$ is the $\mathrm{NR} z$-radius expressed in $\mathrm{nm}$. The other parameters are chosen as $R_{\mathrm{z}, \min [\mathrm{nm}]}=8, R_{\mathrm{z}, \max [\mathrm{nm}]}=440$, and $n_{0}=9 \times 10^{13} \mathrm{~cm}^{-3}$; the corresponding total density and molar concentration are calculated as $n_{\text {tot }}=2 \times 10^{12} \mathrm{~cm}^{-3}$ and $c_{\text {tot }}=3.4 \times 10^{-9} \mathrm{M}$. The distribution function (4) was specially chosen to produce an absorption spectrum that is almost flat in a broad spectral range. Indeed, we see in Figure 4 that the calculated absorption is strong and nearly constant in the range $400 \mathrm{~nm}$ to $6 \mu \mathrm{m}$. Since we assume that the distribution of NRs is continuous and obeys exactly the above law, this is certainly a model case. At the same time, the density of the NR mixture in this case is a realistic number. Now we remove NRs with the sizes in the interval $25<r_{z[\mathrm{~nm}]}<35$ from this mixture and obtain the following distribution function

$$
\begin{aligned}
\delta n_{\mathrm{NR}, \text { window }}\left(r_{z}\right)= & n_{0} \frac{1}{r_{z[\mathrm{~nm}]}^{2.6}}\left(\theta\left(r_{z[\mathrm{~nm}]}-35\right)\right. \\
& \left.+\theta\left(25-r_{z[\mathrm{~nm}]}\right)\right)
\end{aligned}
$$

The corresponding gap in the distribution of NR length is in the interval $50 \mathrm{~nm}<c<70 \mathrm{~nm}$. In Figure 3, we illustrate the model of collection of NRs from which we remove NRs with certain sizes $(50 \mathrm{~nm}<c<70 \mathrm{~nm})$. Figure 4 gives now the distribution $\delta n_{\mathrm{NR} \text {,window }}$ and the calculated optical density for NRs with $a=12 \mathrm{~nm}$. We see an excellent transmission window in the spectrum. This sharp and well-defined transmission window appears because the individual Au NRs exhibit very narrow plasmon peaks. The transmission window takes place in the interval $780 \mathrm{~nm}<\lambda<975 \mathrm{~nm}$, which corresponds to the 
plasmonic wavelengths of the NRs with the lengths $c=50$ and $70 \mathrm{~nm}$. This case shows the principle to create a narrow window in the transmission spectra of plasmonic composites.

Discrete Collection of NRs. From the above model case, we now move to a more realistic case of mixture of NRs with a discrete distribution of sizes. Namely we take a composite of 12 sizes of NRs and 2 sizes of NPs (Figure 5). This composite gives an optical window at $\sim 900 \mathrm{~nm}$. Spherical nanoparticles are used to "close" the absorption spectrum in the interval $400-600 \mathrm{~nm}$. One NP size is small, $12 \mathrm{~nm}$, and another is large, $150 \mathrm{~nm}$. To calculate the extinction of the large-size NPs, we used the full electromagnetic Mie theory ${ }^{23}$ because these NPs are of relatively large sizes. The optical density of the NRNP composite is given now by the sum

$$
\mathrm{OD}=\frac{L_{\text {path }}}{\ln [10]} \sum_{i} n_{i} \cdot \sigma_{i}
$$

where the index $i$ labels the kind of nanocrystals (NR and NP) and $\sigma_{i}$ is the corresponding cross section. Taking a sufficiently long optical path of the NR-NP composite medium, we obtain a spectrum of transmission with a well-defined optical window at about $900 \mathrm{~nm}$ (Figure 5). In Supporting Information we also show the contributions of individual nanocrystals to the total optical density. This particular composition was chosen to create both a wide and nearly flat absorption spectrum in the region $400 \mathrm{~nm}$ to $1.5 \mu \mathrm{m}$ and a narrow transparency window at about $900 \mathrm{~nm}$. In this composition, small Au NPs can be substituted by semiconductor quantum dots (QDs) with an appropriate band gap. For example, CdTe QDs strongly absorb in the region $\lambda<800 \mathrm{~nm}$ and can also be used as an alternative element of the composite. This case is described in Supporting Information.

NRs with small sizes can be fabricated with the wellestablished colloidal synthesis approach. ${ }^{3,4}$ To demonstrate the transparency window effect, we used NRs with a diameter of 12 $\mathrm{nm}$ and lengths of 44-132 nm. Of course, this effect can be demonstrated with many other collections of NR sizes. The particular NR sizes used by us here are very typical for the colloidal nanocrystals. To realize the transparency window effect, one can think about a few experimental systems: (1) Colloidal NRs that can be dispersed in a liquid solution; (2) NRs composition can be built into a polymer film or coating. For this, among other approaches, one can employ the layer-bylayer deposition technique.

\section{LARGE NONINTERACTING PLASMONIC NANORODS: ELECTROMAGNETIC EFFECTS}

Electromagnetic Properties of Single NRs. Large NRs can be fabricated on a substrate using the lithography. Then, optical properties of NRs can be measured directly on a substrate, or NRs can be released to a liquid solution ${ }^{24-26}$ where the optical absorption can be recorded. As another processing step, NRs in solution can be deposited on a polymer film and then covered with another polymer layer using the standard layer-by-layer technology.

NRs with sizes comparable to the wavelength of light exhibit strong retardation effects. Such nanorods should be calculated numerically. In Figures S4 and S6 we plot the extinctions of nanorods computed numerically by the Finite Element Method (COMSOL). We choose the geometry and sizes feasible for the lithographical nanofabrications and, therefore, the NR dimensions are taken larger than those for the colloidal NRs.

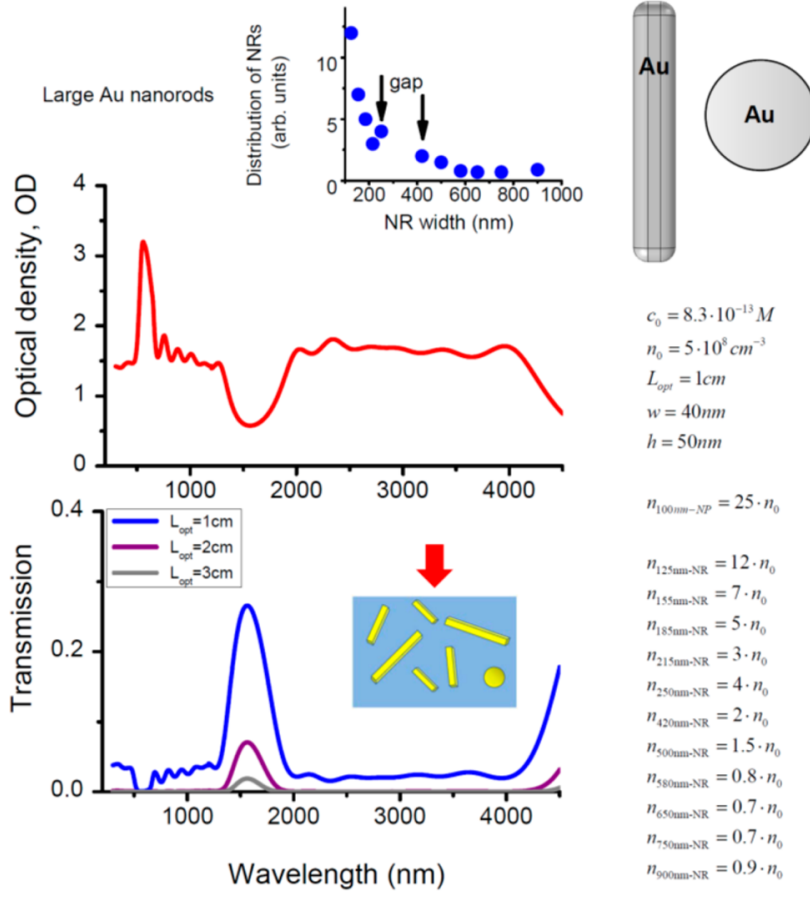

Figure 7. Optical density and transmission of the medium incorporating a collection of large NRs and NPs. On the right-hand side, we list the concentrations of nanocrystals. Insets: Distribution of NR sizes and a model of plasmonic composite medium incorporating both NRs and NPs.

Simultaneously, since we aim at the transparency window effect, we choose relatively small sizes to avoid strong radiative broadening of the L-plasmon peaks. Namely, we take the NR width and height as $w=40 \mathrm{~nm}$ and $h=50 \mathrm{~nm}$. The NR length, $L$, is a variable parameter. First of all, we see that the extinction now has a strong component of scattering and this scattering contribution starts to dominate for large sizes (Figure S4). Another important effect is the radiative broadening. With increase of NR size, the plasmon peak becomes much broader (Figure 6). This is the effect of radiative decay of plasmon. Another observation is that the NR geometry creates typically narrower plasmon peaks compared to the nanodisks (Figure $6 \mathrm{~b}$ ). Therefore, to realize the optical transparency window effect, we prefer to use the NRs. In Supporting Information we give convenient analytical equations for the L-plasmon position and extinction of large lithographic NRs.

Discrete Collections of Large NRs. Large plasmonic NRs, which can be fabricated lithographically, allow us to construct plasmonic media with a narrow transparency window in a much wider spectral range. In principle, one can construct a material with a transparency window in the interval spanning from 700 $\mathrm{nm}$ to $1 \mathrm{~mm}$. We now describe one realization of the NR composition with the optical window around $1500 \mathrm{~nm}$. For this, we choose NR sizes in the interval 125-900 nm and again create a gap in the size distribution. In this case, the gap is taken between 250 and $420 \mathrm{~nm}$. It means that the NRs with the Lplasmon wavelengths around $1500 \mathrm{~nm}$ are not present in the collection. As before, we supplement the NR collection with large spherical $\mathrm{Au}$ NPs that create strong absorption for $\lambda \leq$ $600 \mathrm{~nm}$. Figures 7 and S5 show the calculated spectra.

One interesting observation in the calculated transmission spectra is that the transparency window becomes narrower with an increased optical path because of the nonlinear property of 
a)

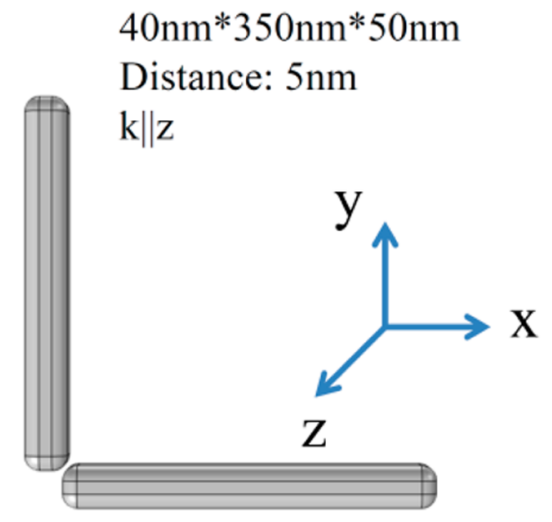

b)

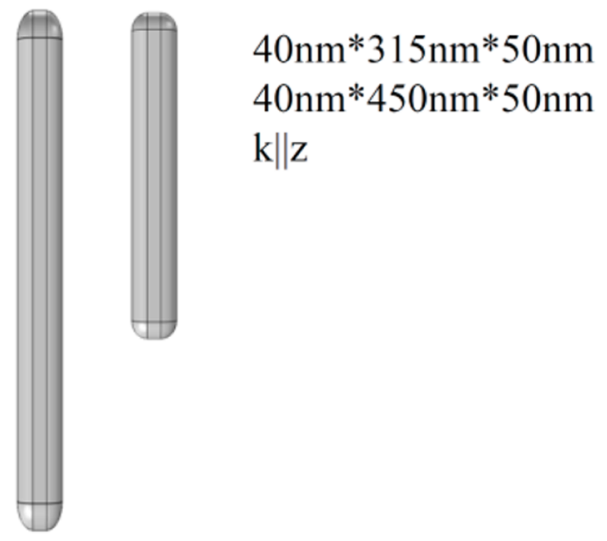

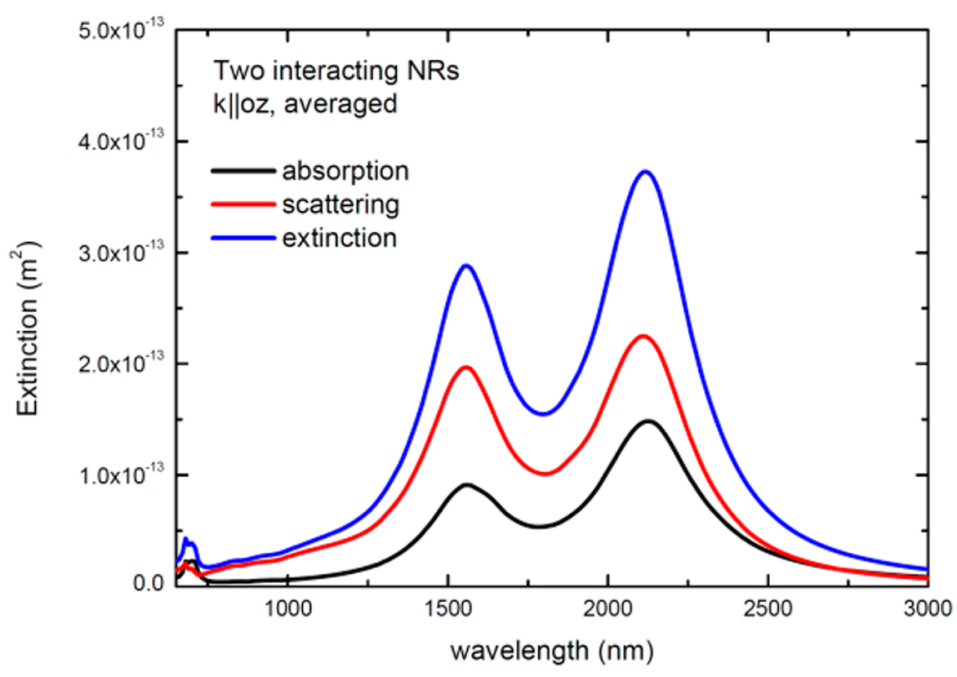

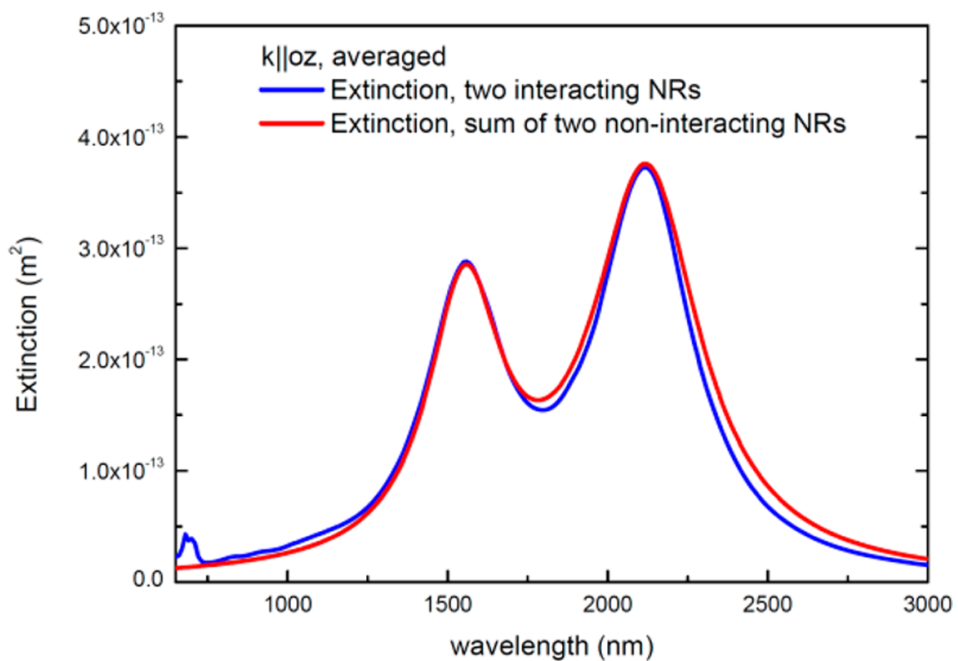

Figure 8. (a) Extinction spectra of a double-NR structure; single NRs in this structure are strongly interacting and the resultant spectrum exhibits a plasmon splitting. (b) Comparison of extinctions for the double-NR structure and the pair of noninteracting NRs. The extinction of the NR pair can be well approximated as a simple sum of extinctions of single NRs with the appropriately chosen lengths. Extinctions were averaged over the polarization of light assuming the normal incidence. Insets show the models.

the exponential function (Figure 7). The same effect can be achieved if we increase the concentration of particles. Therefore, the window effect can be enhanced either with an increased concentration of nanocrystals or by making the optical path longer.

We also should comment on the spectral width and depth of the transparency window in our collections of NRs. These characteristics involve two factors: The broadening of Lplasmon of single NRs and the size-distribution function of a NR ensample. The ultimate width of the transparency window is given by the broadening of L-plasmons of single NRs which includes the Drude and radiative terms. The depth of the transparency window can be increased with increasing the size gap in a NR distribution (inset in Figure 7). By playing with NR distribution functions and single-NR sizes, one can optimize the characteristics of the spectral transparency window in the plasmonic NR compositions.

Interactions between Single NRs. The NR composites discussed above were random collections of NRs and NPs suspended in a solution or built into a polymer matrix. The optical density of such composites was calculated as a linear superposition of extinctions of individual components. This approach assumes that the elements, NRs and NPs, are weakly interacting. We have shown numerically that the interactions can be neglected if the internanocrystal distance in a composite is about or larger than the sizes of components. Supporting Information gives a few examples of interactions between nanocrystals. If the internanocrystal distance is, however, smaller than the lengths of components, the electromagnetic interactions become strong and most likely destroy the window effect. In Figure S6, we show such a case. In the model used in Figure S6, we arrange $17 \mathrm{NRs}$ in an array with a relatively small inter-NR distance. Then, we calculate the total extinction and compare it with the case of linear superposition. Whereas the linear superposition has a transparency window at $\sim 1500 \mathrm{~nm}$, the metamaterial structure, which looks like a pipe organ, does not exhibit the optical window at all. In this structure, strong inter-NR electromagnetic interactions modify the whole spectrum and completely destroy the transparency window effect.

Another interesting question is whether the interactions between single nanocrystals can help to create a narrower 


\section{$40 \mathrm{~nm} * 370 \mathrm{~nm} * 50 \mathrm{~nm}$ \\ $40 \mathrm{~nm} * 230 \mathrm{~nm} * 50 \mathrm{~nm}$ \\ $\mathrm{k} \| \mathrm{z}$, averaged}
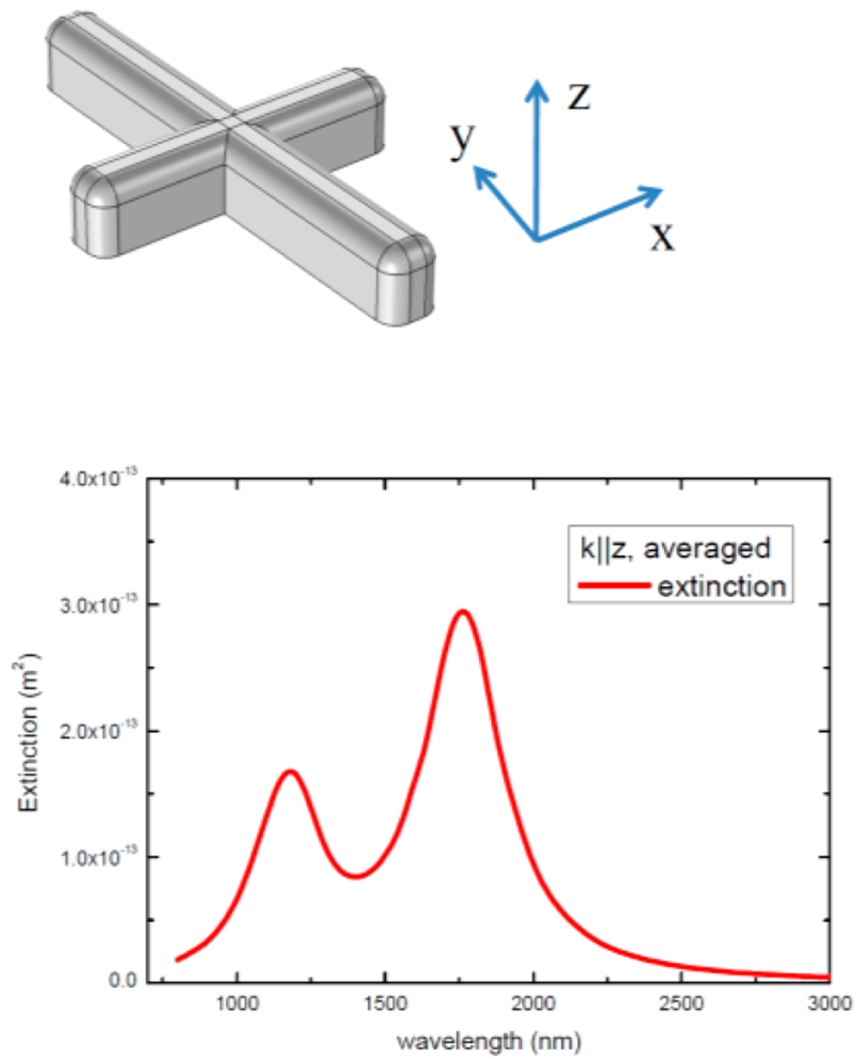

$40 \mathrm{~nm} * 250 \mathrm{~nm} * 50 \mathrm{~nm}$

$40 \mathrm{~nm} * 400 \mathrm{~nm} * 50 \mathrm{~nm}$

Distance: $150 \mathrm{~nm}$

$\mathrm{k} \| \mathrm{z}$, averaged

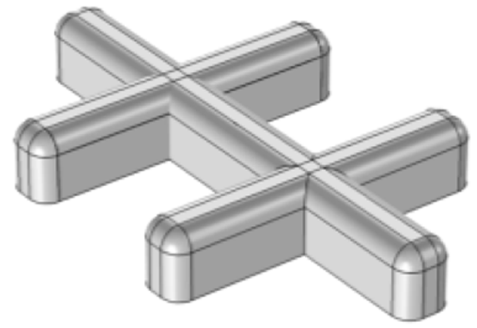

Extinction y.s. $\lambda$

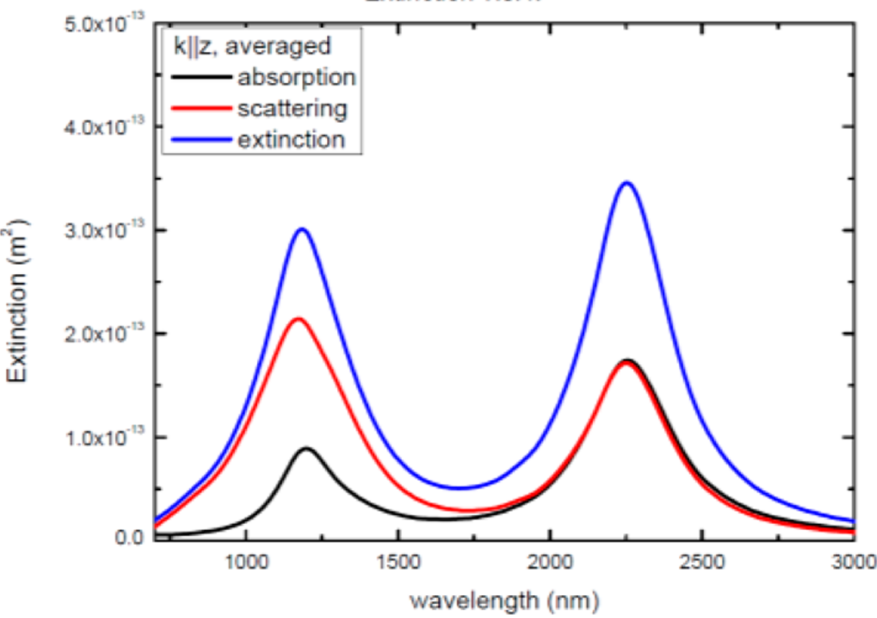

Figure 9. Extinction spectra of simple and two-bar nanocrosses. The two-bar nanocross shows an almost symmetric double-peak structure that is needed for the window effect. The extinctions are calculated for the normal incidence and then averaged over the polarization of light.

transparency window. In a nanostructure composed of two NRs, we expect the emergence of the splitting and the related double-peak spectral structure. The question is whether such a double peak structure can be used for the transparency window effect. Figure 8 shows that the simple NR-NR interaction does not improve the window effect. The window effect of the two interacting NRs is almost identical to the effect created by two independent NRs with appropriately chosen sizes. We should note that this is just one particular case of internanocrystal interaction and there may be other cases when the interactions can be helpful for the transparency window effects.

\section{METASTRUCTURES AND METAMATERIALS}

We now look at more complex structures. The idea is to find more complex elementary blocks that can be more compact and convenient. Such nanocomposite material can occupy less space and also have fewer elements which may simplify a fabrication. We could already see that two interacting NRs do not give much advantage since the window effect is similar to the case of two noninteracting nanocrystals (Figure 8). Additionally, the structure with two perpendicular NRs exhibits an asymmetric double peak spectrum, whereas for materials with a transparency window, we better have elements with symmetric double-peak spectra. For this, we will try now simple and multibar nanocrosses.
Nanocrosses. We aim to find an element that has a symmetric double-peak structure, which can be used for a metamaterial with a transparency window. However, simple nanocrosses give typically asymmetric windows (Figure 9). Therefore, we can try a cross with two perpendicular bars. After the optimization of the design parameters, we show that the two-bar cross can produce a symmetric window. A symmetric window effect appears only for certain, specially chosen geometrical parameters. For example, the two-bar cross (cross 3) with a large interbar distance in Figure 10 produces an asymmetric spectral window since the long-wavelength peak becomes enhanced. The next step is to use the nanocrosses as elements in a metastructure.

Composition of Nanocrosses, Nanorods, and Nanodisks. We now involve three crosses, two NRs, and two nanodisks (NDs). Figure 11 shows the composition. All these elements can be fabricated with the lithography technologies. The height of the nanostructures is taken as $h=50 \mathrm{~nm}$ and the width of the bars in these structures is chosen as $w=40 \mathrm{~nm}$. Three nanocrosses give three double-peak structures with the transparency window in the middle. NRs and NDs block the transmission in the long- and short-wavelength intervals, correspondingly. The resulting spectrum exhibits strong extinction in the interval $400 \mathrm{~nm}$ to $4.5 \mu \mathrm{m}$ and a transparency window centered at $1660 \mathrm{~nm}$. 


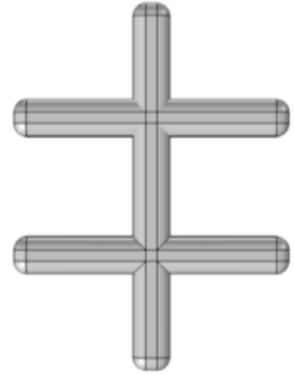

Cross 1:

$40 \mathrm{~nm} * 300 \mathrm{~nm} * 50 \mathrm{~nm}$

$40 \mathrm{~nm} * 400 \mathrm{~nm} * 50 \mathrm{~nm}$

Distance: $150 \mathrm{~nm}$
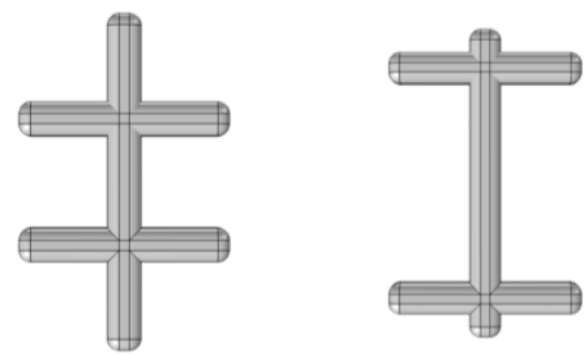

Cross 2:

$40 \mathrm{~nm} * 250 \mathrm{~nm} * 50 \mathrm{~nm}$

$40 \mathrm{~nm} * 400 \mathrm{~nm} * 50 \mathrm{~nm}$

Distance: $150 \mathrm{~nm}$
Cross 3:

$40 \mathrm{~nm} * 250 \mathrm{~nm} * 50 \mathrm{~nm}$

$40 \mathrm{~nm} * 400 \mathrm{~nm} * 50 \mathrm{~nm}$

Distance: $300 \mathrm{~nm}$

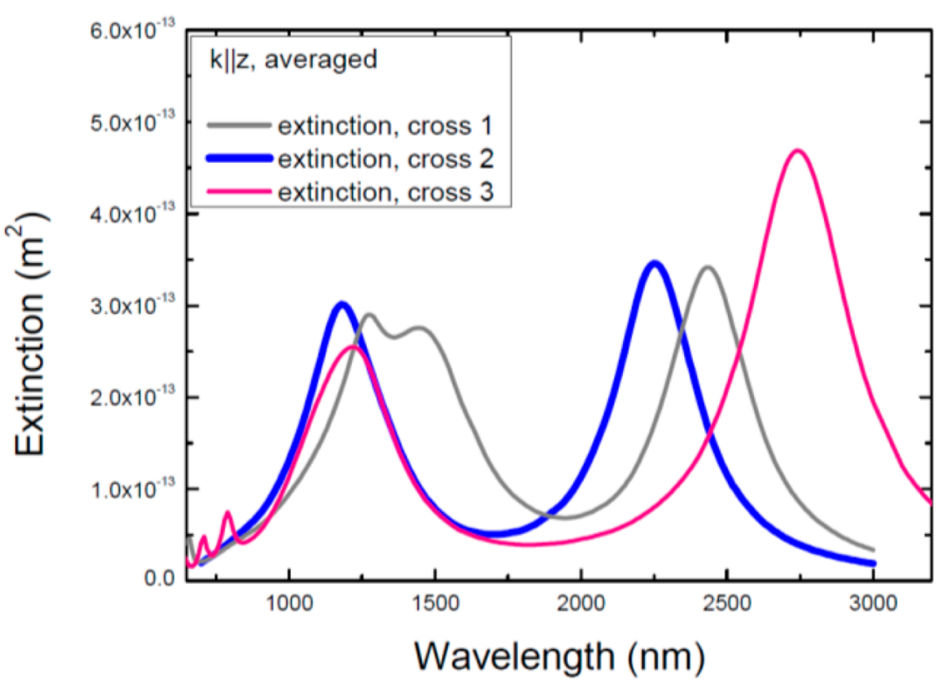

Figure 10. Extinction spectra of two-bar nanocrosses with different parameters. The two-bar nanocross in the center exhibits an almost symmetric window effect. Again, the extinctions are calculated for the normal incidence and then averaged over the orthogonal polarization states of light.

The optical medium with the spectral transparency window can also be fabricated as a planar meta-material on a substrate. Figure 12 shows this case. The distances between the nanocrystals in this metastructure are long enough to avoid interference between the elements. The distances can be found in Figure S8. In addition, in this metastructure, we suppress the interference effects by orienting the nanocrystals. To do this, we arrange the elements in a way to avoid quasi-resonant interactions between the elements. For example, we orient the two-bar and three-bar crosses perpendicular to each other. Similarly, we make the three-bar and four-bar crosses perpendicular as well. Therefore, three interacting nanocrosses in our structure do not show prominent effects of interaction. Also, two long NRs are placed at a large distance from each other and on the common line. The nanodisks are well separated and do not interact much. We confirm that the interactions in our structure are weak by the direct numerical COMSOL calculations shown in Supporting Information. By positioning and orienting the elements in a certain way, we can avoid strong interactions between nanocrystals and a distortion of the window effect.
We have performed a number of calculations of interacting nanocrystals and found the following simple rules to construct metastructures with weak interactions. (1) We orient two quasiresonant elongated elements (NRs or nanocrosses) perpendicular to each other. Then, the L-plasmon resonances do not interact due to the symmetry. (2) Long NRs with very strong plasmon resonances better be placed on a common line. (3) We place the elements that are resonant or quasi-resonant as far as possible from each other on a substrate. (4) Spherical and cylindrical elements should have interparticle distances that are about their sizes. The logistics behind these rules is to reduce the interference effects in a metastructure using the geometrical approach.

We also should make a few final comments on fabrication methods for the described metastructures. Thin substrates (glass or polymer) with metastructures can be randomly dispersed in a medium (liquid, polymer or air) or oriented as parallel multilayers in a polymer matrix (Figure 12b). The resulting transmission spectrum of such system shows a strong transparency window effect (Figure 12b). This is another possibility to realize an optical material with both a strong broadband extinction spectrum and a narrow transmission 


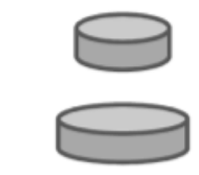

$100 \mathrm{~nm}$ ( 8 disks) $200 \mathrm{~nm}$ (3 disks)

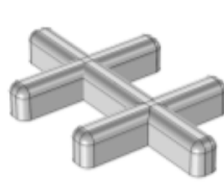

$40 \mathrm{~nm} * 250 \mathrm{~nm} * 50 \mathrm{~nm}$ $40 \mathrm{~nm}^{*} 400 \mathrm{~nm}^{*} 50 \mathrm{~nm}$ Distance: $150 \mathrm{~nm}$

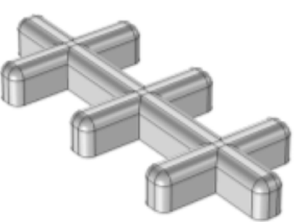

$40 \mathrm{~nm} * 200 \mathrm{~nm} * 50 \mathrm{~nm}$ $40 \mathrm{~nm}^{*} 500 \mathrm{~nm}^{*} 50 \mathrm{~nm}$ Distance: $150 \mathrm{~nm}$

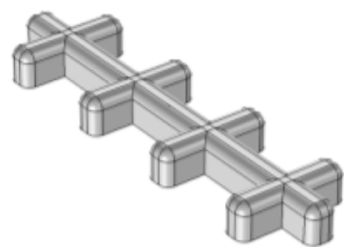

$40 \mathrm{~nm} * 160 \mathrm{~nm} * 50 \mathrm{~nm}$ $40 \mathrm{~nm}^{*} 600 \mathrm{~nm} * 50 \mathrm{~nm}$ Distance: $150 \mathrm{~nm}$

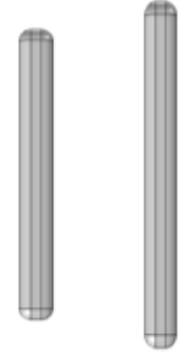

$40 \mathrm{~nm} * 800 \mathrm{~nm} * 50 \mathrm{~nm}$ $40 \mathrm{~nm} * 950 \mathrm{~nm} * 50 \mathrm{~nm}$

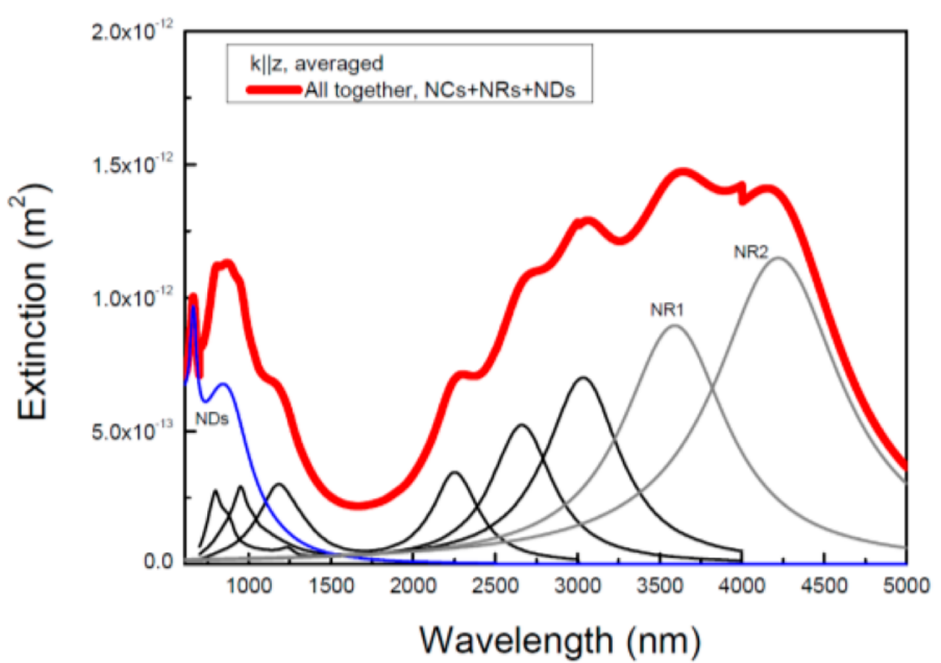

Figure 11. Extinction spectrum of the composition incorporating three nanocrosses, two nanorods, and two nanodisks. All elements are made of gold. Insets show the geometries of the elements.

a)

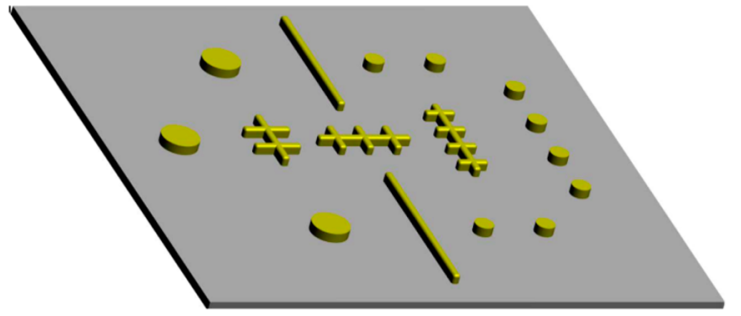

b)
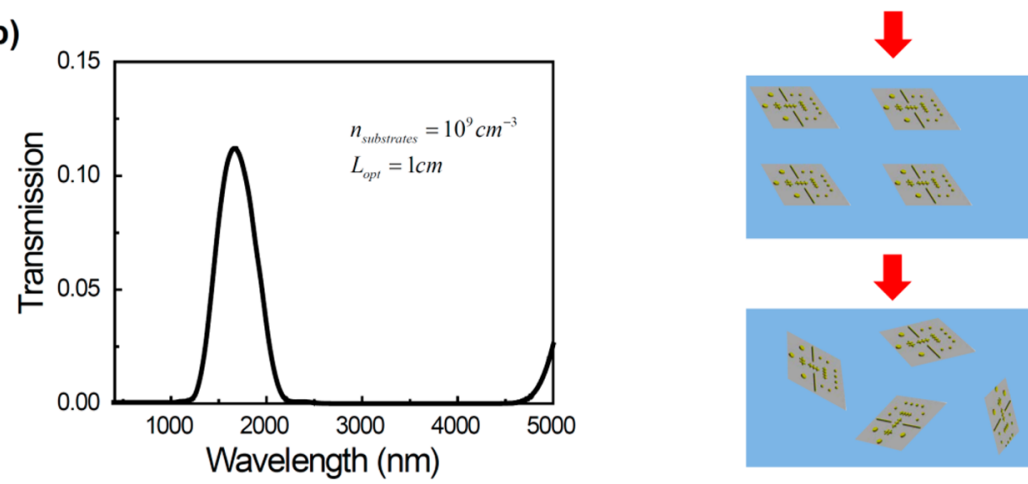

Figure 12. (a) Model of planar plasmonic metastructure featuring a transparency window. (b) Calculated transmission spectrum of a solution with dispersed metastructures having random orientations. Insets show two models: A layered medium based on a polymer matrix and a liquid solution with dispersed metastructures.

window. Since the lithography allows us to fabricate nanorods and nanowires with an almost arbitrary length (of course, within the reasonable limits), the proposed optical metamaterials may have transparency windows within a wavelength interval from $600 \mathrm{~nm}$ to $1 \mathrm{~mm}$.

\section{CONCLUSIONS}

We have proposed and described a novel class of nanostructured materials with very peculiar optical properties. This set of nanomaterials strongly attenuates light across a very 
broad spectrum, but exhibits a narrow window of transmission. The transparency window can be designed to be sharp or arbitrary broad. The principle to construct these optical media is based on narrow plasmonic peaks of metal nanorods and nanocrosses. The optical medium is constructed as a mixture of elements with a specially designed distribution of sizes. Nanomaterials with a transparency window can be fabricated by using both the conventional colloidal synthesis and the lithography technologies. These materials can be realized as dispersions of single nanocrystals in liquid or as twodimensional metastructures on a substrate. Optical media with a transparency window, which can be made at a chosen wavelength, can be in the forms of dispersion solutions, polymer films, and coatings. Using plasmonic nanorods and nanocrystals, the transmission window can be designed at any particular wavelength in the interval from $400 \mathrm{~nm}$ to tens of microns and further to the infrared.

\section{ASSOCIATED CONTENT}

\section{S Supporting Information}

This information includes analytical derivations for optical properties of plasmonic nanorods and computational results for optical spectra of several compositions, nanocrosses, and metastructures. This material is available free of charge via the Internet at http://pubs.acs.org.

\section{AUTHOR INFORMATION}

\section{Corresponding Author}

*E-mail: govorov@ohiou.edu.

\section{Notes}

The authors declare no competing financial interest.

\section{ACKNOWLEDGMENTS}

We thank Z. Fan for help with the computations. H.Z. and A.O.G. were supported by the U.S. Army Research Office under Grant Number W911NF-12-1-0407 and by the Volkswagen Foundation (Germany). Use of the Computing Cluster at the Center for Nanoscale Materials was supported by the U.S. Department of Energy, Office of Science, Office of Basic Energy Sciences, under Contract No. DE-AC0206CH11357. H.V.D. gratefully acknowledges support from NRF-RF-2009-09 and NRF-CRP-6-2010-2, as well as TUBA and ESF EURYI.

\section{REFERENCES}

(1) Maier, S. A. Plasmonics: Fundamentals and Applications; Springer: N.Y., 2007.

(2) Novotny, L.; Hecht, B. Principles of Nano-Optics; Cambridge Univ. Press: Cambridge, U.K., 2006.

(3) Complex-Shaped Metal Nanoparticles. Bottom-Up Syntheses and Applications; Sau, T. K., Rogach, A. L., Eds.; Wiley-VCH: Weinheim, 2013.

(4) Link, S.; El-Sayed, M. A. Spectral properties and relaxation dynamics of surface plasmon electronic oscillations in gold and silver nanodots and nanorods. J. Phys. Chem. B 1999, 103, 8410-8426.

(5) Dondapati, S. K.; Sau, T. K.; Hrelescu, C.; Klar, T. A.; Stefani, F. D.; Feldmann, J. Label-free biosensing based on single gold nanostars as plasmonic transducers. ACS Nano 2010, 4 (11), 6318-6322.

(6) Shelby, R. A.; Smith, D. R.; Schultz, S. Experimental verification of a negative index of refraction. Science 2001, 292, 77-79.

(7) Luk'yanchuk, B.; Zheludev, N. I.; Maier, S. A.; Halas, N. J.; Nordlander, P.; Giessen, H.; Chong, C. T. The Fano resonance in plasmonic nanostructures and metamaterials. Nat. Mater. 2001, 9, $707-715$.
(8) Fan, J. A.; Wu, C.; Bao, K.; Bao, J.; Bardhan, R.; Halas, N. J.; Manoharan, V. N.; Nordlander, P.; Shvets, G.; Capasso, F. Selfassembled plasmonic nanoparticle clusters. Science 2010, 328, 11351138.

(9) Verellen, N.; Sonnefraud, Y.; Sobhani, H.; Hao, F.; Moshchalkov, V. V.; Dorpe, P. V.; Nordlander, P.; Maier, S. A. Fano resonances in individual coherent plasmonic nanocavities. Nano Lett. 2009, 9, 16631667.

(10) Shvets, G.; Urzhumov, Y. A. Engineering the electromagnetic properties of periodic nanostructures using electrostatic resonances. Phys. Rev. Lett. 2004, 93 (243902), 1-4.

(11) Wiederrecht, G. P.; Wurtz, G. A.; Hranisavljevic, J. Coherent coupling of molecular excitons to electronic polarizations of noble metal nanoparticles. Nano Lett. 2004, 4, 2121-2125.

(12) Zhang, W.; Govorov, A. O.; Bryant, G. W. Semiconductor-metal nanoparticle molecules: hybrid excitons and non-linear Fano effect. Phys. Rev. Lett. 2006, 97 (146804), 1-4.

(13) Shah, R. A.; Scherer, N. F.; Pelton, M.; Gray, S. K. Ultrafast reversal of a Fano resonance in a plasmon-exciton system. Phys. Rev. B 2013, 88 (075411), 1-7.

(14) Zhang, J.; Tang, Y.; Lee, K.; Ouyang, M. Tailoring light-matterspin interactions in colloidal hetero-nanostructures. Nature 2010, 466, 91-95.

(15) Zhang, S.; Genov, D. A.; Wang, Y.; Liu, M.; Zhang, X. Plasmoninduced transparency in metamaterials. Phys. Rev. Lett. 2008, 101 (047401), 1-4.

(16) Liu, N.; Langguth, L.; Weiss, T.; Kästel, J.; Fleischhauer, M.; Pfau, T.; Giessen, H. Plasmonic analogue of electromagnetically induced transparency at the Drude damping limit. Nat. Mater. 2009, 8, $758-762$.

(17) Biswas, S.; Duan, J.; Nepal, D.; Park, K.; Pachter, R.; Vaia, R. Plasmon induced transparency in the visible wavelengths enabled by self-assembled metamaterials. Nano Lett. 2013, 13, 6287-6291.

(18) Ebbesen, T. W.; Lezec, H. J.; Ghaemi, H. F.; Thio, T.; Wolff, P. A. Extraordinary optical transmission through sub-wavelength hole arrays. Nature 1998, 391, 667-669.

(19) Landau, L. D.; Lifshitz, E. M. Electrodynamics of Continuous Media; Pergamon: N. Y., 1960.

(20) van de Hulst, H. C. Light Scattering by Small Particles; Dover Publications: N.Y., 1981.

(21) Johnson, P. B.; Christy, R. W. Optical constants of the noble metals. Phys. Rev. B 1972, 6, 4370-4379.

(22) Vial, A.; Grimault, A.-S.; Macías, D.; Barchiesi, D.; de La Chapelle, M. L. Improved analytical fit of gold dispersion: Application to the modeling of extinction spectra with a finite-difference timedomain method. Phys. Rev. B 2005, 71 (085416), 1-7.

(23) http://icb.u-bourgogne.fr/nano/manapi/saviot/mie/index.en. html.

(24) Lee, J.; Hasan, W.; Stender, C. L.; Odom, T. W. Pyramids: a platform for designing multifunctional plasmonic particles. Acc. Chem. Res. 2008, 41, 1762-1771.

(25) Mark, A. G.; Gibbs, J. G.; Lee, T.-C.; Fischer, P. Hybrid nanocolloids with programmed three-dimensional shape and material composition. Nat. Mater. 2013, 12, 802-807.

(26) Yeom, B.; Zhang, H.; Zhang, H.; Park, J.; Kim, K.; Govorov, A. O.; Kotov, N. A. Chiral plasmonic nanostructures on achiral nanopillars. Nano Lett. 2013, 13, 5277-5283. 